SVU- International Journal of Veterinary Sciences, 2 (2): 45-51, 2019.

\title{
Prospects and limitations of physiologically-based pharmacokinetic modelling for cross- species extrapolation
}

\section{Lars Kuepfer*1, 2}

${ }^{1}$ Research and Development, Pharmaceuticals, Systems Pharmacology and Medicine, Bayer AG, Germany, ${ }^{2}$ Institute of Applied Microbiology, RWTH Aachen, Germany.

Abstract

The transition from experimental findings in animal models to clinical applications in human patients is a key challenge in pharmacology and toxicology. To date, this step still inhibits a significant level of uncertainty explaining amongst others the continuously high attrition rates in pharmaceutical development programs. Computational modelling bears the promise to support cross-species extrapolation through mechanistic descriptions of relevant physiological processes. In this review, the benefits and limitations of computational approaches for clinical translation are discussed and the needs for future applications are outlined. A particular focus is laid on the differentiation between pharmacokinetics and pharmacodynamics. While the former determines drug exposure in plasma or specific tissues, the latter describes the resulting response, i.e. the therapeutic outcome or an adverse event. Based on a previous study it is argued that the main challenges for cross-species extrapolation is genetic divergence between different animal models and humans which will require novel inter-disciplinary concepts for clinical translation in the future.

Keywords: Cross-species extrapolation, Inter-species extrapolation, PBPK modelling, Pharmacology, Toxicology.

DOI: $10.21608 /$ svu.2019.14193.1020

Received: June 30, 2019

Accepted: July 2, 2019

Published: July 3, 2019

*Corresponding Author: Lars Kuepfer

E-mail: lars.kuepfer@bayer.com

Citation: Kuepfer, Prospects and limitations of physiologically-based pharmacokinetic modelling for cross-species extrapolation. SVU-IJVS 2019, 2 (2): 45-51.

Copyright: (C) Kuepfer. This is an open access article distributed under the terms of the creative common attribution license, which permits unrestricted use, distribution and reproduction in any medium provided the original author and source are created.

Competing interest: The authors have declared that no competing interest exists.

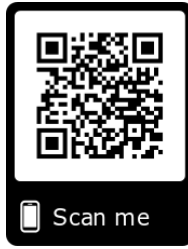


Animal experiments are still routinely used in pharmacology and toxicology. In pharmaceutical development, animal models are a mandatory milestone before a new compound is administered to humans in clinical phases I - III. The main objectives in the preclinical phase are patient safety, i.e. to identify a non-toxic dose for first-in-man studies, and to explore the to-be-expected therapeutic window of a novel compound. Today, $31 \%$ of all pharmaceutical development programs are discontinued in the preclinical phase, while $46 \%$ of all projects in phase I are stopped (Paul et al. 2010). The majority of these project terminations are due to safety reasons $(82 \%$ in the preclinical phase and $62 \%$ in phase I, respectively) (Cook et al. 2014). Likewise, it has been estimated that only about $43 \%$ of toxic side effects in humans are correctly predicted (Hartung 2009). It can thus be concluded that animal experiments are in a significant number of cases not informative with regard to drug safety. This clearly indicates the need for novel rational approaches to translate results from animal experiments to human patients. Given an increasingly high ethical (and scientific) standard in animal welfare, it is mandatory to design tailor-made animal experiments to provide reliable insights which cannot be obtained otherwise. Note that such claims date back to the 1950s when 3R principles (3R: reducer, refine, replace) for animal experiments were formulated first (Russell and Burch 1959).

A substantial contribution to the establishment of $3 \mathrm{R}$ principles in pharmacology and toxicology can be expected from computational modeling (Hartung et al. 2017). This is because pharmacokinetic (PK) models provide an important possibility to quantify plasma exposure to xenobiotic compounds. For cross-species extrapolation of PK parameters in compartmental PK models allometric scaling is usually applied. Such approaches are based on exponential regression equations to predict parameters in another species. However, it should be noted that this approach is largely datadriven with uncertain translational power. This was shown in a systematic study of 102 small molecule drugs where an average prediction error of $254 \%$ was calculated for allometric scaling of pharmacokinetic parameters from rats, dogs, and monkeys to humans (Ward and Smith 2004).

An alternative approach to compartmental models is physiologicallybased pharmacokinetic (PBPK) models (Jones et al. 2009; Kuepfer et al. 2016). PBPK models are based on systems of ordinary differential equations and include a large amount of prior physiological information regarding the physiology of an organism. In particular, organs and the connecting vascular circulation are explicitly represented in PBPK models. Physiological processes underlying drug ADME (ADME: absorption, distribution, metabolism and excretion) can hence be described mechanistically. PBPK therefore allows a unique opportunity to simulate time-concentration profiles in blood and different tissues. Moreover, enzymemediated metabolisation as well as transport processes can be explicitly considered in PBPK models (Meyer et al. 2012), Table (1). Due to the large level of physiological detail represented in PBPK models, they are ideally suited for extrapolation from a basic reference model to novel scenarios (e.g. different patient subgroups, different preclinical species or 
different doses and administration schemes, etc.).

In the simplest case, extrapolations in PBPK models only require modifications of a single physiological model parameter such as a reduction in liver clearance capacity in hepatically impaired patients. PBPK modelling in humans has for example been successfully used to describe the functional impact of different phenotypes of ADME proteins on drug pharmacokinetics in patients through modulation of catalytic activities (Cordes et al. 2016; Lippert et al. 2012). Likewise, PBPK models have been used to quantify the difference in endosomal clearance of various monoclonal antibody variants through adjustments of the binding affinities to the neonatal $\mathrm{Fc}$ receptor in cynomolgus monkeys (Niederalt et al. 2018). More extended extrapolations with simultaneous alterations of multiple physiological model parameters involve simulations of pediatric drug pharmacokinetics (Maharaj and Edginton 2014) or elderly patients (Schlender et al. 2018). The use of PBPK modelling in clinical studies requires well-designed analysis plans for model qualification and statistical analyses. The application of PBPK modelling in pharmaceutical development programs is strongly encouraged by regulatory agencies to support decision making through dedicated computational simulations.

In the light of the growing acceptance of PBPK modelling in pharmaceutical research is a self-evident and tempting conclusion to apply PBPK modelling for cross-species extrapolations. This would significantly support the translation of results from preclinical studies to first-inman studies and to later clinical phases. Surprisingly, there are still few scientific papers applying PBPK modelling for extrapolations to different mammalian species (106 hits for a PubMed search for
"PBPK" AND ("cross-species" OR "interspecies" OR "interspecies") before June 2019). A significant number of these works stems from toxicology each discussing very specific case studies and applications for single compounds (Campbell et al. 2017). In an attempt to assess the basic possibilities and limitations of PBPK-based cross-species extrapolation, a comparative study was performed in which the impact of different degrees of prior information on the accuracy of cross-species extrapolation has been systematically evaluated (Thiel et al. 2015). In this study, the benefit of using different domains of prior, species-specific input was systematically assessed in mice and humans for PBPK models of ten marketed small molecules (molecular weight 194.2 - $1202.6 \mathrm{~g} / \mathrm{mol})$. This input included: (1) species-specific physiology, (2) plasma protein binding, (3) enzyme and transporter kinetics and (4) tissue-specific gene expression and was either considered exclusively or in different permutation. Notably, the drug-specific metabolism of each of the ten compounds was of comparable complexity with one active process. Hence, the contribution of ADME proteins to the overall drug PK could be well described, quantified and differentiated. The key outcome of this analysis was that species-specific physiology is essential to achieve accurate simulation results when extrapolating a PBPK model from a reference to a target species. In addition, plasma protein binding, kinetic rate constants and gene expression data could be shown to synergistically contribute to the quality of simulation outcomes if they are considered simultaneously (Thiel et al. 2015). A plausible interpretation of these findings is that species-specific physiology alone largely determines passive processes of drug PK. This is because the basic volume of distribution is thus determined by 
setting organ volumes, organ surface areas or organ blood flow rates as well as the connecting vascular circulation. Also, passive distribution of drugs within different tissues can be directly quantified in PBPK models from physicochemical properties of a compound (Jones et al. 2009; Kuepfer et al. 2016). Plasma protein binding, ADME gene expression as well as enzyme and transporter kinetics then describe the overlaying active ADME processes in addition to species-specific physiology and passive distribution processes.

One challenge for the application of computational models for cross-species extrapolation is genetic divergence in ADME genes including the cytochrome p450 protein family between different mammalian species (Vamathevan et al. 2013). This also holds for species differences in drug transporter expression and their activity, which is still poorly understood (Chu et al. 2013). As an

immediate consequence, ADME
parameters are difficult to translate between different animal models. Evaluating the homology of an orthologous ADME gene in different species is one possibility to assess the functional comparability of an enzyme or drug transporter in different animal species. However, a full in vitro characterisation of active processes is necessary for the support of cross-species translation by targeted in vitro-in vivo extrapolation (Thiel et al. 2015). This is in particular important in the case of limited sequence similarity in orthologous genes. An even more sophisticated approach are humanised animal models (Nadeau and Auwerx 2019). It remains to be shown whether scaling of experimental data from such models through species-specific PBPK models could further improve the accuracy of cross-species extrapolations as such accounting for physiological differences.

Table (1): Processes in drug PK/PD involving drug distribution and molecular interactions.

\begin{tabular}{l|l|l}
\hline & Pharmacokinetics (PK) & Pharmacodynamics (PD) \\
\hline Physiology and passive processes & $\begin{array}{l}\text { + drug ADME and drug } \\
\text { distribution }\end{array}$ & \\
\hline $\begin{array}{l}\text { Molecular interactions and active } \\
\text { processes }\end{array}$ & $\begin{array}{l}\text { - ADME proteins and } \\
\text { drug transporters }\end{array}$ & $\begin{array}{l}\text { - drug interactions with } \\
\text { endogenous networks }\end{array}$ \\
\hline
\end{tabular}

Finally, it should be noted that any downstream pharmacodynamics (PD) effect will further increase species-specific differences due to the multitude of on- and off-target drug-interactions since PBPK models merely describe physiological ADME processes underlying drug disposition in the body. PD effects are therefore a result of the xenobiotic compound interfering with the limited set of ADME proteins and with several endogenous networks. To account for such resulting species-specific divergence in drug responses remains a key challenge in pharmacology and toxicology especially since some analyses are only possible in animal models (Ghallab et al. 2016; Ghallab et al. 2019; Schenk et al.). To 
translate findings from animal models to human patients and to simultaneously reduce the number of animal sacrifices will require novel inter-disciplinary concepts for clinical translation in the future encompassing both targeted experimental design and computational modelling.

\section{Conflict of interest statement}

The authors declare that they have no conflict of interest.

\section{References}

Campbell JL, Jr Andersen ME, Van Landingham C, Gentry R, Jensen E, Domoradzki JY, Clewell HJ 3rd (2017). Refinement of the oral exposure description in the cyclic siloxane PBPK model for rats and humans: Implications for exposure assessment. Toxicology Letters, 279 Suppl 1: 125-135. doi: 10.1016/j.toxlet.2017.04.002

Chu X, Bleasby K, Evers R (2013). Species differences in drug transporters and implications for translating preclinical findings to humans. Expert Opinion on Drug Metabolism and Toxicology, 9(3): 237-252. doi: 10.1517/17425255.2013.741589

Cook D, Brown D, Alexander R, March R, Morgan P, Satterthwaite G, Pangalos MN (2014). Lessons learned from the fate of AstraZeneca's drug pipeline: a fivedimensional framework. Nature Reviews. Drug Discovovery, 13(6): 419-431. doi: 10.1038/nrd4309

Cordes H, Thiel C, Aschmann HE, Baier V, Blank LM, Kuepfer L. (2016). A Physiologically Based Pharmacokinetic Model of Isoniazid and Its Application in Individualizing Tuberculosis
Chemotherapy. Antimicrobial Agents and Chemotherapy, 60(10): 6134-6145. doi: 10.1128/AAC.00508-16

Ghallab A, Celliere G, Henkel SG, Driesch D, Hoehme S, Hofmann U, Hengstler JG (2016). Model-guided identification of a therapeutic strategy to reduce hyperammonemia in liver diseases. Journal of Hepatology, 64(4): 860871. doi: 10.1016/j.jhep.2015.11.018

Ghallab A, Hofmann U, Sezgin S, Vartak $\mathrm{N}$, Hassan R, Zaza A, Reif R (2019). Bile Microinfarcts in Cholestasis Are Initiated by Rupture of the Apical Hepatocyte Membrane and Cause Shunting of Bile to Sinusoidal Blood. Hepatology, 69(2): 666-683. doi: 10.1002/hep.30213

Hartung T (2009). A toxicology for the 21 st century--mapping the road ahead. Toxicological Sciences: an official journal of the Society of Toxicology, 109(1), 18-23. doi: 10.1093/toxsci/kfp059

Hartung T, FitzGerald RE, Jennings $\mathrm{P}$, Mirams GR, Peitsch MC, RostamiHodjegan A, Sturla SJ (2017). Systems Toxicology: Real World Applications and Opportunities. Chemical Research in Toxicology, 30(4): 870-882. doi: 10.1021/acs.chemrestox.7b00003

Jones HM, Gardner IB, Watson KJ (2009). Modelling and PBPK simulation in drug discovery. The AAPS journal, 11(1): 155-166.

Kuepfer L, Niederalt C, Wendl T, Schlender JF, Willmann S, Lippert, J, Teutonico D (2016). Applied Concepts in PBPK Modeling: How to Build a PBPK/PD Model. CPT Pharmacometrics and Systems 
Pharmacology, 5(10): 516-531. doi: $10.1002 / \mathrm{psp} 4.12134$

Lippert J, Brosch M, von Kampen O, Meyer M, Siegmund HU, Schafmayer C, Kuepfer L (2012). A mechanistic, model-based approach to safety assessment in clinical development. CPT Pharmacometrics and System Pharmacology, 1: e13. doi: 10.1038/psp.2012.14

Maharaj AR, Edginton AN (2014). Physiologically based pharmacokinetic modeling and simulation in pediatric drug development. CPT Pharmacometrics and System Pharmacology, 3: e150. doi: 10.1038/psp.2014.45

Meyer M, Schneckener S, Ludewig B, Kuepfer L, Lippert J (2012). Using expression data for quantification of active processes in physiologically based pharmacokinetic modeling. Drug Metabolism and Disposition: the Biological Fate of Chemicals, 40(5): 892-901. doi: 10.1124/dmd.111.043174

Nadeau JH, Auwerx J (2019). The virtuous cycle of human genetics and mouse models in drug discovery. Nature Reviews. Drug Discovery, 18(4): 255-272. doi: 10.1038/s41573-0180009-9

Niederalt C, Kuepfer L, Solodenko J, Eissing T, Siegmund HU, Block M, Lippert J (2018). A generic whole body physiologically based pharmacokinetic model for therapeutic proteins in PK-Sim. Journal of Pharmacokinetics and Pharmacodynamics, 45(2): 235257. doi: 10.1007/s10928-0179559-4

Paul SM, Mytelka DS, Dunwiddie CT, Persinger $\mathrm{CC}$, Munos $\mathrm{BH}$,
Lindborg SR, Schacht AL (2010). How to improve $\mathrm{R} \& \mathrm{D}$ productivity: the pharmaceutical industry's grand challenge. Nature Reviews. Drug Discovery, 9(3): 203-214. doi: 10.1038/nrd3078

Russell WMS, Burch RL (1959). The principles of humane experimental technique. London, UK: Methuen, 238.

Schenk A, Ghallab A, Hofmann U, Hassan R, Schwarz M, Schuppert A, Kuepfer L. Physiologically-based modelling in mice suggests an aggravated loss of clearance capacity after toxic liver damage Sciientific Reports, accepted.

Schlender JF, Teutonico D, Coboeken K, Schnizler K, Eissing T, Willmann S, Stass H (2018). A Physiologically-Based

Pharmacokinetic Model to Describe Ciprofloxacin Pharmacokinetics Over the Entire Span of Life. Clinical Pharmacokinetics, 57(12): 16131634. doi: 10.1007/s40262-0180661-6

Tang and Mayersohn J Pharm Sci. 2006 Aug;95(8):1783-99

(https://www.ncbi.nlm.nih.gov/pub med/16795013)

Thiel C, Schneckener S, Krauss M, Ghallab A, Hofmann U, Kanacher T, Kuepfer L (2015). A systematic evaluation of the use of physiologically based pharmacokinetic modeling for cross-species extrapolation. Journal of Pharmaceutical Sciences, 104(1): 191-206. doi: 10.1002/jps. 24214

Vamathevan JJ, Hall MD, Hasan S, Woollard PM, $\mathrm{Xu} \mathrm{M}$, Yang Y, Sanseau P (2013). Minipig and beagle animal model genomes aid 
species selection in pharmaceutical discovery and development. Toxicology and Applied Pharmacology, 270(2): 149157. doi: 10.1016/j.taap.2013.04.007

Ward KW, Smith BR (2004). A comprehensive quantitative and qualitative evaluation of extrapolation of intravenous pharmacokinetic parameters from rat, dog, and monkey to humans. I. Clearance. Drug Metabolism and Disposition, 32(6): 603-611. doi: 10.1124/dmd.32.6.603 